Journal of Animal and Veterinary Advances 11 (16): 2845-2851, 2012

ISSN: $1680-5593$

(C) Medwell Journals, 2012

\title{
Comparison of Adipose Tissue Derived Stem Cells from Different Subcutaneous Adipose Tissue Depots of Canine
}

\author{
${ }^{1,2}$ Jing Yang, ${ }^{3}$ Xiangang Cai, ${ }^{1,2}$ Tao Song, ${ }^{1,2}$ Yongjun Chen, ${ }^{1,2} \mathrm{Hui}$ Chen, \\ ${ }^{1,2} \mathrm{P}$ an $\mathrm{Wu}$ and ${ }^{1,2}$ Congxin Huang \\ ${ }^{1}$ Department of Cardiology, Renmin Hospital of Wuhan University, \\ ${ }^{2}$ Cardiovascular Research Institute, Wuhan University, 430060 Wuhan, P.R. China \\ ${ }^{3}$ Department of Cardiology, The Thirteen Hospital of Wuhan City, 430100 Wuhan, P.R. China
}

\begin{abstract}
Adipose-derived Stem Cells (ASCs) have great potential in the field of tissue engineering. There is evidence that human ASCs from different depots of adipose tissue may produce different characteristics. Canine is a large and important animal model for human diseases which has led to interest in the isolation and characterization of canine ASCs. Canine Adipose tissue derived mesenchymal Stem Cells (cASCs) also have been shown to possess the proliferation and multi-differentiate capacity. The main goal of this research was to compare the proliferation capacity, phenotypes, apoptosis susceptibility, differentiation capacity and gene transfection efficiency of cASCs obtained from different depots (superficial abdominal, hip, thigh, dorsal and inguinal) of subcutaneous adipose tissue. The proliferation rate of cASCs from abdominal and hip subcutaneous adipose tissue were higher than other depots. cASCs from abdominal subcutaneous adipose tissue had higher capacity to differentiate into adipogenic lineages than other depots. However, cASCs from dorsal subcutaneous adipose tissue had the highest capacity to differentiate into Osteogenic lineages among all depots. Regarding to the cardiomyogenic differentiation, the results showed that there was no differences among cASCs from different subcutaneous adipose tissue. Apoptosis susceptibility was demonstrated to be lowest in the superficial abdominal depot. In conclusion, proliferation, differentiate capacity and sensitivity to apoptosis of cASCs were linked to anatomic subcutaneous adipose tissue depots whereas there were no significant differences in the expression of surface antigens and gene transduction efficiency.
\end{abstract}

Key words: Subcutaneous adipose tissue, mesenchymal stem cells, canine adipose tissue derived stem cells, tissue engineering, transduction

\section{INTRODUCTION}

Adipose tissue may represent a potential source of adult stem cells for tissue engineering applications (Schaffler and Buchler, 2007). It can be obtained in large quantities, under local anesthesia and with minimal discomfort. Adipose-derived Stem Cells (ASCs) have great potential in the field of tissue engineering. Scientists compared the biological characteristics of ASCs from Subcutaneous (SC) and $\mathrm{OM}$ adipose tissues and found that different depots of adipose tissue may produce ASCs with different characteristics (Van Harmelen et al., 2004). These differences may be due to the regional variation in cell types and inherent properties of ASCs. Toyoda et al. (2009) demonstrated that the higher differentiation capacity of ASCs from SC adipose tissue which suggested that $\mathrm{SC}$ adipose tissue be more suitable cell source for regenerative medicine than $\mathrm{OM}$ adipose tissue. Based on anatomic location, the huamn body can be derived into various depots of subcutaneous adipose tissue. Schipper observed that the grow and differentiate ability of human ASCs varies from various SC depots.

However, there are species differences in the properties of adipose-derived MSCs. The importance of the dog as a large animal model of human diseases has led to interest in the isolation and characterization of canine ASCs. Some studies have been done to demonstrate the potential of canine ASCs for tissue regeneration and gene dilivery (Cui et al., 2007; Lim et al., 2010). But few researches were done to address the differences of cASCs from different $\mathrm{SC}$ depots. We must recognize variations in subcutaneous depots by studying their behavior. For this purpose, researchers compared the proliferation capacity, phenotypes, differentiation capacity and gene transfection efficiency of cASCs obtained from different depot of subcutaneous adipose tissue (Superficial abdominal, gluteal, thigh, dorsal and inguinal). The aims of this study were to identify differences in

Corresponding Author: Congxin Huang, Cardiovascular Research Institute, Wuhan University, Division of Cardiology, Renmin Hospital of Wuhan University, Wuhan University, 430060 Wuhan, P.R. China 
cASCs biology from 5 different subcutaneous anatomic locations. This study will help select adipose tissue sites most suitable for tissue engineering therapies. By comparing the characteristics of cASCs from various subcutaneous depots, researchers may be able to identify an ideal depot to yield cASCs for tissue engineering as well as understand biologic differences of cASCs from different regions adipose tissue of the dogs.

\section{MATERIALS AND METHODS}

Collection of adipose tissue: Canine adipose tissue was $(<1 \mathrm{~cm} \mathrm{3)} \mathrm{obtained} \mathrm{from} \mathrm{subcutaneous} \mathrm{region} \mathrm{(Superficial-}$ abdominal, hip, thigh, dorsal and inguinal). The study protocol was approved by the Institutional Animal Care and Use Ethical Committees of Wuhan University. About 7-9 months old canines were obtained for the experiments.

Isolation and culture of cASCs: After anesthetizated with sodium pentobarbital (30 $\mathrm{mg} \mathrm{kg}^{-1}$ intraperitoneal injection), adipose tissue from different SC depots of dog was washed with sterile Phosphate Buffered Saline (PBS) and was digested by $1 \mathrm{mg} \mathrm{mL}^{-1}$ collagenase Type I (Invitrogen) was performed for $45 \mathrm{~min}$ at $37^{\circ} \mathrm{C}$ during permanent shaking. The cell suspension was centrifuged at $1000 \mathrm{~g}$ for $10 \mathrm{~min}$. The cells were washed and then cultivated in complete medium containing L-DMEM (Gibco) supplemented with 10\% New Bovine Serum (NBS, hyclone) and $1 \%$ penicillin/streptomycin (Invitrogen) in a $37^{\circ} \mathrm{C}$ incubator with a $5 \% \mathrm{CO}_{2}$ atmosphere. After cultured for $72 \mathrm{~h}$, the medium was removed to discard unattached cells and then refed with fresh medium. When the cells reached $80 \%$ of confluence, they were detached from the culture flask using $0.25 \%$ trypsin containing $1 \mathrm{mM}$ EDTA (Sigma) for further analyses.

Measurement of population doubling time: Approximately, $2500 \mathrm{cASCs}$ at 5 passage from each depot were seeded in triplicate into 96 well plates to develop growth curves, $10 \mu \mathrm{L}$ of cell counting kit-8 (Beyotime, China) were added to each well and incubated for $3 \mathrm{~h}$ at $37^{\circ} \mathrm{C}$. The optical density values were determined in triplicate at a test wavelength of $450 \mathrm{~nm}$ and reference wavelength of $630 \mathrm{~nm}$. The doubling time was calculated during the logarithmic phase of the growth curve. The time of population doublings was calculated using the equation:

$$
\mathrm{Td}=\mathrm{T} \times \frac{\log 2}{\log \left(\frac{\mathrm{ODt}}{\mathrm{OD} 0}\right)}
$$

$\mathrm{T}=$ The time of the logarithmic phase of the growth curve

ODO $=$ The $O D$ value after seeding

ODt $=$ The OD value at the end of the logarithmic growth phase

Flow cytometry analysis: $\operatorname{cASCs}\left(1 \times 10^{6}\right)$ at passage 5 from each depot were suspended in $500 \mu \mathrm{L}$ PBS containing $20 \mathrm{ng} \mathrm{mL}^{-1}$ Fluorescein Isothiocyanate (FITC)coupled antibodies against CD29, CD44, CD90, CD14 and CD34 (Biolegend). After incubated for $30 \mathrm{~min}$ at $4^{\circ} \mathrm{C}$, the cells were washed with PBS and resuspended in $1 \mathrm{~mL}$ PBS for analysis. Cell fluorescence was evaluated by flow cytometry in a FACS Calibur instrument (Becton Dickinson) and data were analyzed by using CellQuest software (Becton Dickinson).

Apoptosis susceptibility: Approximately 30,000 cASCs from each depot were seeded in triplicate in a 24 well plate. After $24 \mathrm{~h}$, the media was removed and fresh media with a solution of $1.0 \mu \mathrm{M}$ staurosporine was added to each well. A cell death detection Enzyme-Linked Immunosorbent Assay-Plus kit (Roche) was analyzed $24 \mathrm{~h}$ after addition of the staurosporine. The absorbance is measured at $405 \mathrm{~nm}$ and the susceptibility to the apoptotic agent is detected.

Adipogenic differentiation: Subconfluent (80\%) cASCs from each depot were plated at the seeding density of 10,000 cells $\mathrm{cm}^{-2}$ in 6 well plates using adipogenic induction medium consisting of $1 \mathrm{mM}$ dexamethasone, $0.5 \mathrm{mM}$ 3-isobutyl-1-methyl-xanthine, $10 \mu \mathrm{g} \mathrm{mL}-1$ recombinant human insulin, $100 \mathrm{mM}$ indomethacin and $10 \%$ FCS. Adipogenic differentiation was confirmed by the formation of neutral lipid-vacuoles stainable with Oil Red-O (Sigma). The adipogenic cultures were fixed in $4 \%$ paraformaldehyde for at least $1 \mathrm{~h}$ and stained with fresh Oil Red-O solution for $2 \mathrm{~h}$. The Oil Red-O solution was prepared by mixing three parts of stock solution $(0.5 \%$ in isopropanol; Sigma) with two parts of water and filtering through a $0.2 \mu \mathrm{m}$ filter. After being stained, the cultures were washed three times and the number of colonies positive with Oil Red-O was counted. The percentage of differentiated cells was determined by counting differntiated and undifferentiated cells in 5 randomly selected areas $\left(\mathrm{mm}^{2}\right)$ under the microscope.

Osteogenic differentiation: Osteogenic differentiation was induced for 3 weeks in osteogenic medium consisting of complete medium supplemented with $0.1 \mu \mathrm{M}$ dexamethasone, $50 \mu \mathrm{M}$ ascorbate-2-phosphate and $10 \mathrm{mM} \beta$-glycerophosphate (Pittenger et al., 1999). To 
assess the osteolastic phenotype, the positive ratio of Alkaline Phosphatase (ALP) staining was tested (Beyotime, China).

Cardiomyogenic differentiation: For cardiomyogenic induction, subconfluent (80\%) cASCs were induced with 5-azacytidine for $72 \mathrm{~h}$ after cells had been cultured for 4 weeks, cTnI with IgG-FITC (Biolegend) was used to examine cardiomyogenesis.

Gene transduction: Gene transduction efficiency of cASCs from each depot was examined using a adenovirus vector which was an E1-deleted, replication-deficient adenoviral vector with EGFP reporter gene driven by a CMV promoter (pACCMVGFP). Cells were infected with viral vectors for $2 \mathrm{~h}$ at $37^{\circ} \mathrm{C}$ and EGFP expression was analyzed after infection by flow cytometry.

Statistical analysis: Data was presented as mean \pm standard deviation. Comparisons among cASCs isolated from the different depots of adipose tissue were performed by using the nonparametric Kruskal-Wallis exact test. Differences were considered significant at $\mathrm{p}<0.05$. The SPSS Software Package 13.0 was used for the statistical tests.

\section{RESULTS AND DISCUSSION}

Morphorlogy of cASCs: Non-adherent cells were removed after $72 \mathrm{~h}$. After an initial lag time of 2-3 days, cells entered proliferative phase, reaching $80 \%$ confluence within 14 days. Adherent cells were observed after $72 \mathrm{~h}$ in culture and cells quickly generated a monolayer of fibroblastic-like morphology (Fig. 1a-c). The morphology of cASCs was similar in the five different SC depots analyzed.

Cell surface antigen analysis: Researchers examined the expression of CD29, CD44, CD73, CD90, CD105, CD14 and CD34 of cASCs from five different depots. At passage 5, the majority of cASCs expressed CD29, CD 44 and CD90 while CD14 and CD34 were negative. The expression levels of cell-surface antigen analyzed had no significant differences among cASCs from five different depots. The results were shown in Table 1.

Table 1: Comparison of the expression of surface proteins analyzed by flow cytometry each value is the mean percentage of at least three experiments \pm standard deviation

\begin{tabular}{lccccc}
\hline Depots & CD29 & CD44 & CD90 & CD14 & CD34 \\
\hline Abdominal & $95.3 \pm 3.1$ & $89.2 \pm 5.4$ & $92.1 \pm 4.2$ & $0.9 \pm 0.4$ & $1.9 \pm 0.5$ \\
Hip & $96.7 \pm 2.4$ & $88.7 \pm 3.6$ & $93.2 \pm 2.2$ & $1.1 \pm 0.5$ & $1.3 \pm 0.4$ \\
Thigh & $95.2 \pm 1.8$ & $86.5 \pm 4.4$ & $91.7 \pm 1.4$ & $1.3 \pm 0.4$ & $1.6 \pm 0.7$ \\
Dorsal & $96.0 \pm 1.2$ & $90.1 \pm 3.1$ & $90.6 \pm 2.1$ & $1.2 \pm 0.1$ & $1.4 \pm 0.2$ \\
Inguinal & $94.6 \pm 0.6$ & $88.3 \pm 3.2$ & $92.0 \pm 2.3$ & $1.0 \pm 0.3$ & $1.5 \pm 0.4$ \\
\hline
\end{tabular}

Growth kinetics of cASCs: To compare the proliferation rate of cASCs from different subcutaneous adipose tissue depots from the same donor, the cells at passage 5 were collected. The proliferation rates of cASCs from abdominal region and hip subcutaneous adipose tissue were obviously faster than other depots.

The average doubling time of cASCs in the exponential growing phase for abdominal region and hip subcutaneous adipose tissue was $28.15 \pm 4.12$ and $30.17 \pm 3.49 \mathrm{~h}$ (Fig. 2), respectively. However, the proliferation rate and doubling time were not significantly different among cASCs from thigh, dorsal and inguinal depots.

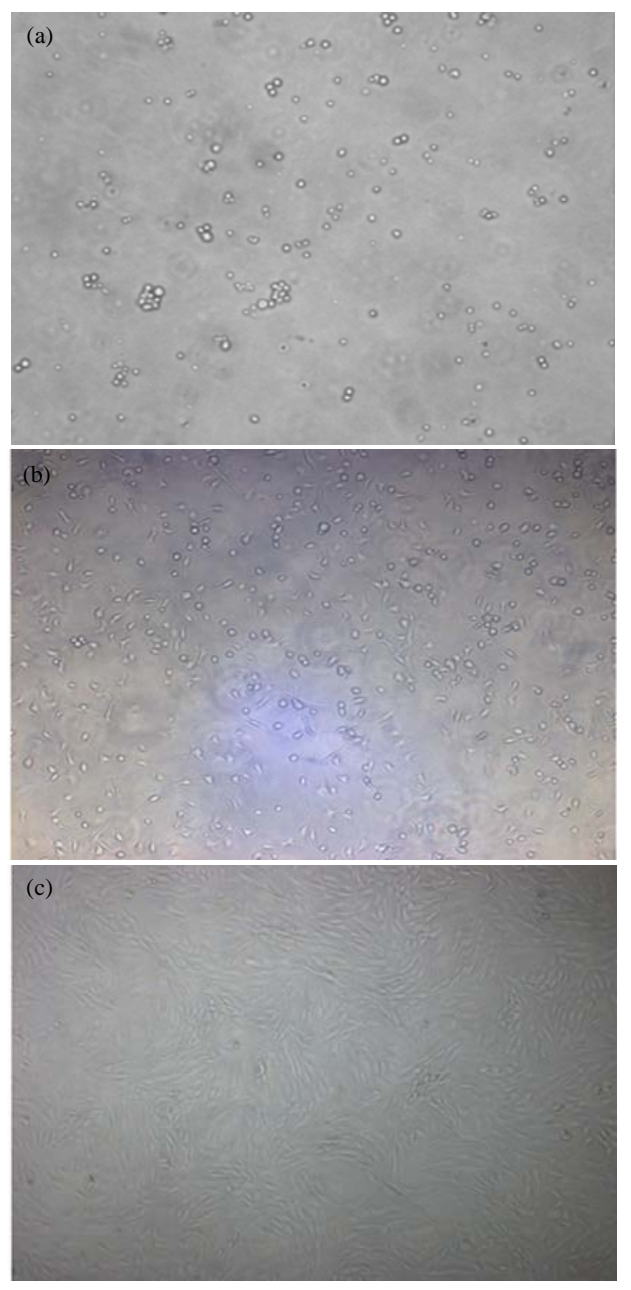

Fig. 1: Morphology of cASCs from subcutaneous abdominal adipose tissue cultured in vitro. Cells were plated in culture flask in $72 \mathrm{~h}$; a) The adherent cells grew into spindle-shaped cells after being plated for 4 days; b) cASCs reached $80 \%$ confluence at the 7 th day; c) Scar bars: $20 \mu \mathrm{m}$ 
Apoptosis susceptibility: Researchers found that there were variations on apoptosis susceptibility among cASCs from different SC depots (Fig. 3a-e). Compared with other depots, the superficial abdominal depot was statistically significantly lest susceptible to an apoptotic stimulus. Apoptosis susceptibility was demonstrated to be lowest in the cASCs from superficial abdominal depot (Fig. 3f).

Adipogenic potential: Many lipid vesicles were observed in the cells after being induced for 3 weeks (Fig. 4a). However, there were few lipid vesicles in control group (Fig. 4d). The Oil Red-O colony positive rate was calculated by dividing the number of Oil Red-O positive

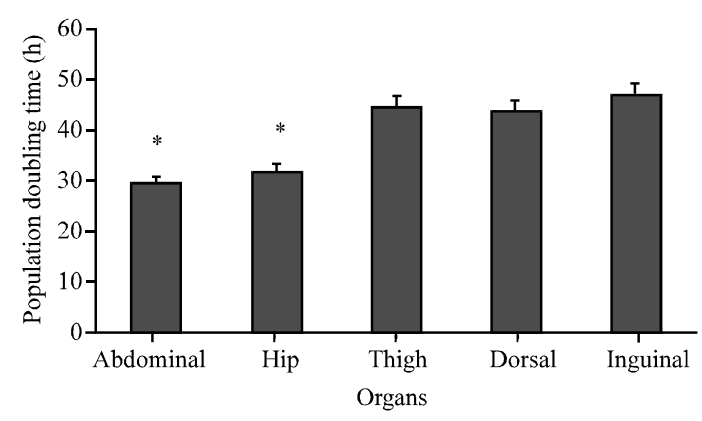

Fig. 2: Analysis of population-doubling time of cASCs from different subcutaneous adipose tissue depots at passage 5. Data were obtained from five donors per adipose tissue depot and averaged from triplicate wells. Population-doubling time of cASCs from abdominal and hip depots were shorter than those from other depots $\left({ }^{*} \mathrm{p}<0.05\right)$ cells by the number of total cells of the same culture. The Oil Red-O positive rate in cASCs from abdominal was higher than other depots $(p<0.05)$ indicating the differences in adipogenic potential. The percentage of cells staining positive for Oil Red-O was $54.3 \pm 5.1 \%$ of cASCs from abdominal depot (Fig. 5).

Osteogenic potential: To compare the osteogenic differentiation capacity of cASCs from different depots, all cASCs were cultured in osteogenic medium for 3 weeks. After osteogenic differentiation induction, ALP staining was test (Fig. 4b and e). Results showed that there were differences in the osteogenic differentiation capacity among cASCs. The positive ratio of ALP staining in cASCs from dorsal was higher than other depots $(p<0.05)$. (Fig. 5).

Cardiomyogenic potential: After induced by 5-AZA for $72 \mathrm{~h}$, cASCs were culture in complete medium for 4 weeks. Morphology changes and spontaneous cellular beating were not seen. Several cells showed green fluorescence for cTnI (Fig. 4c). The positive ratios had no obvious differences among cASCs from five different SC depots (Fig. 5).

Gene transduction: Transduction efficiency (EGFP expression) was analyzed by flow cytometry after transduction for $72 \mathrm{~h}$. cASCs from 5 different SC depots were successfully transduced with adenovirus with no observed cytotoxicity at the multiplicities of infection used. About $75 \%$ of cASCs were EGFP positive

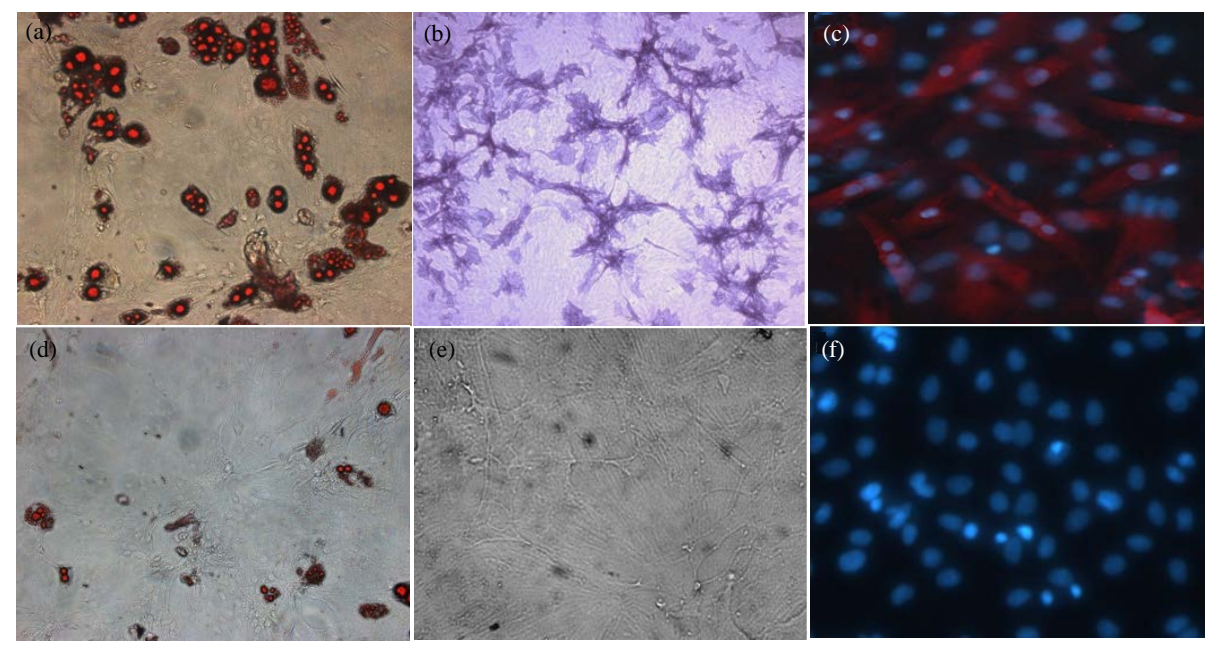

Fig. 3: Apoptosis susceptibility among cASCs from different SC depots. a) Abdominal; b) Hip; c) Thigh; d) Dorsal and e) Inguinal. Compared with other depots, f) apoptosis susceptibility was demonstrated to be lowest in the cASCs from superficial abdominal depot $\left({ }^{*} \mathrm{p}<0.01\right)$ 

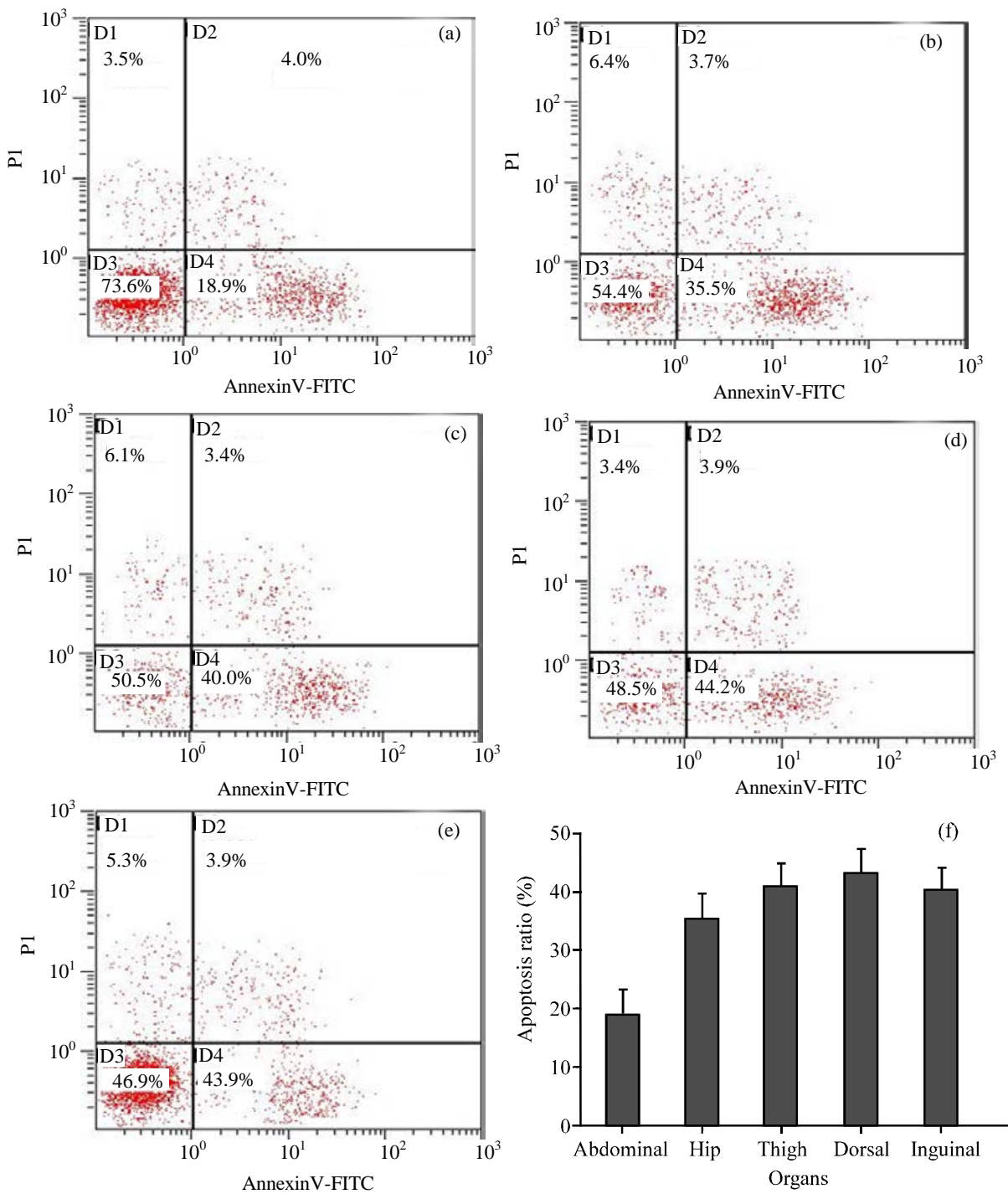

Fig. 4: Differentiation potential of cASCs at passage 5. About 3 weeks after initial induction, adipogenic differentiation was confirmed by Oil Red-O staining of lipid droplets present throughout the cytoplasm; a) Osteogenic differentiation was demonstrated by ALP staining; b) cASCs differentiated into cardiomyocytes by 5-AZA, cardiomyocyte were cTnI positive; c) In contrast, uninduced cells were negative for Oil Red-O; d) ALP; e) cTnI and f) Staining. Scar bars: $50 \mu \mathrm{m}$

and the expression of EGFP were not decline after passaging. The results revealed that there were no differences in adenoviral transduction efficiency among cASCs from different SC depots.

Mesenchymal Stem Cells (MSCs) are a very important adult stem cell population with potential applications in regenerative medicine. Adipose tissue may represent a potential source of adult stem cells for tissue engineering applications. Adipose tissue can be obtained in large quantities, under local anesthesia and with minimal discomfort. Zuk et al. $(2002,2001)$ were the first to describe the isolation and characterization of human stem cells derived from adipose tissue. Adipose tissue-derived mesenchymal Stem Cells (ASCs) have many qualities that render these cells well suited for tissue engineering applications such as proliferationand multidifferentiation ability. Other groups reported successful isolation of adipose stem cells from other mammals such as mouse (Yamamoto et al., 2007), rat (Lopez and Spencer, 2011), rabbit (Torres et al., 2007), equine (Vidal et al., 2007), porcine (Williams et al., 2008) and bovine (Ren et al., 2010). Neupane et al. (2008) demonstrated the isolation of canine ASCs with extensive proliferation potential and multilineage differentiation ability. Futhermore, they compared proliferation potential of cASCs among subcutaneous, omental and inguinal fat 


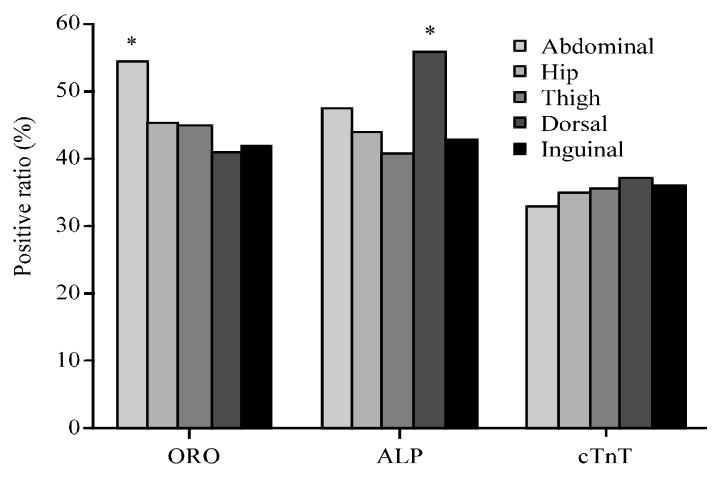

Biochemical compounds

Fig. 5: Comparison of adipogenic, osteogenic and cardiogenic differentiation ratio of $\mathrm{cASCs}$ from different subcutaneous adipose tissue depots. ORO, adipogenesis, Oil Red-O staining; ALP, osteogenesis, ALP staining; cTnI, cardiogenesis, immuofluorescent staining. ${ }^{*} \mathrm{p}<0.05$ compared with other groups

depots from canines. They showed considerable variation from different sites with fat yielding the greatest numbers of cells.

In this study, researchers compared the characterization of canine ASCs from subcutaneous adipose tissue collected from superficial abdominal, hip, thigh, dorsal and inguinal region of 1 year old dogs. Researchers examined the effect of different subcutaneous depots on the basic properties (proliferation, differentiation and apoptosis susceptibility) of cASCs. Researchers showed that the morphologies of cASCs from SC adipose tissue were heterogeneous with large colonies consisting of small spindle-shaped cells and small colonies comprising large polygonal cells. These data are consistent with previous reports from canine adipose tissue-derived mesenchymal stem cells (Lim et al., 2010; Martinello et al., 2011; Spencer et al., 2011).

Flow cytometry showed that the majority of cASCs are of mesenchymal origin. Researchers also observed that cASCs can be maintained in vitro for extended periods with stable population doubling time. cASCs can be differentiated in vitro into adipogenic, chondrogenic, osteogenic and myogenic cells in the presence of lineage-specific induction factors.

The proliferation potential of ASCs might be influenced by species, the region of tissue collection, age, cell density during plating and media formulation. Schipper et al. (2008) have compared the characterization of adipose-derived stem cells isolated from different depots humans and found that there were no statistically difference among the depots included upper arm, medial thigh, trochanteric and both superficial and deep abdominal. Fraser et al. (2007) reported that human adipose tissue from hip yielded 2.3 fold more ASCs than abdominal and the cells from hip had more potency to differentiate into osteogeninc lineages. The results showed that the proliferation rates of cASCs from abdominal region and hip subcutaneous adipose tissue were obviously faster than other depots. The differences among the results and other reports may be due to species difference.

Under appropriate culture conditions, cASCs were induced to differentiate into osteoblast, adipocyte and cardiomyogenic lineages. In agreement with other groups, researchers found that differences in differentiate ability of different subcutaneous adipose tissue depots. cASCs from hip and abdominal adipose tissue have more capacity to differentiate into adipocyte than from thigh, dorsal and inguinal depots. Results also showed that there were differences in the osteogenic differentiation capacity among cASCs. Researchers also found the differentiate ratio into osteoblast in cASCs from dorsal was higher than other depots. However, the positive ratios of cTnI had no obvious differences among cASCs from five different $\mathrm{SC}$ depots.

In previous studies, Kelly et al. (1999) have showed that adipos stem cells from superficial abdominal depot was least susceptibla to apoptosis when compared with those from the omental depot. Researchers also found that apoptosis susceptibility was lowest in the superficial abdominal depot which was consistent with the reports by Kelly.

Fluorescence microscopic imaging and flow cytometry revealed that the expression ratioes of EGFP were stable after cASCs transduction and there were no differences in adenoviral transduction efficiency among cASCs from different SC depots. The stable EGFP expression after cASCs transduction represents a great advantage in preclinical studies.

Large animal models such as the canine, more faithfully mimic human pathologies. The characterization of cASCs represents a valuable tool for in vitro and in vivo preclinical evaluation. This study may provide important insight into the biology and therapeutic potential of cASCs and may form an increased understanding of the tools needed to successfully engineer adipose tissue. However, as we all known, there are species differences in the properties of ASCs so, the results in an study in a dog model might not be the same in the human case. Therefore, the studies in dogs should be cautiously interpreted when researchers extrapolates them to human. The mechanism underlying these differences should be explored in future studies. 


\section{CONCLUSION}

The results of this study indicate that there are regional differences in proliferation and differentiation capacity among subcutaneous were observed. Canine adipose tissue may also contain multipotent cells and represent an important stem cell source both for preclinical studies. These findings also have potential relevance to future canine tissue engineering and regenerative medical therapies. Suggesting the importance of selection of the harvest depot if one considers to use adipose tissue as a cell sources for regenerative medicine.

\section{ACKNOWLEDGEMENT}

This study was supported by the grants from the National Natural Science Foundation of China (No.: 30700312).

\section{REFERENCES}

Cui, L., B. Liu, G. Liu, W. Zhang and L. Cen et al., 2007. Repair of cranial bone defects with adipose derived stem cells and coral scaffold in a canine model. Biomaterials, 28: 5477-8486.

Fraser, J.K., I. Wulur, Z. Alfonso, M. Zhu and E.S. Wheeler, 2007. Differences in stem and progenitor cell yield in different subcutaneous adipose tissue depots. Cytotherapy, 9: 459-467.

Kelly, I.E., T.S. Han, K. Walsh and M.E. Lean, 1999. Effects of a Thiazolidinedione compound on body fat and fat distribution of patients with type 2 diabetes. Diabetes Care, 22: 288-293.

Lim, J.H., L. Boozer, C.L. Mariani, J.A. Piedrahita and N.J. Olby, 2010. Generation and characterization of neurospheres from canine adipose tissue-derived stromal cells. Cell. Reprogram, 12: 417-425.

Lopez, M.J. and N.D. Spencer, 2011. In vitro adult rat adipose tissue-derived stromal cell isolation and differentiation. Methods Mol. Biol., 702: 37-46.

Martinello, T., I. Bronzini, L. Maccatrozzo, A. Mollo and M. Sampaolesi et al., 2011. Canine adipose-derivedmesenchymal stem cells do not lose stem features after a long-term cryopreservation. Res. Vet. Sci., 91: $18-24$.

Neupane, M., C.C. Chang, M. Kiupel and V. YuzbasiyanGurkan, 2008. Isolation and characterization of canine adipose-derived mesenchymal stem cells. Tissue Eng. Part A, 14: 1007-1015.

Pittenger, M.F., A.M. Mackay, S.C. Beck, R.K. Jaiswal and R. Douglas et al., 1999. Multilineage potential of adult human mesenchymal stem cells. Science, 284: $143-147$.
Ren, Y., H. Wu, Y. Ma, M. Cang, R. Wang and D. Liu, 2010. Isolation, cultivation and identification of adipose-derived stem cell in bovines. Sheng $\mathrm{Wu}$ Gong Cheng Xue Bao, 26: 1645-1651.

Schaffler, A. and C. Buchler, 2007. Concise Review: Adipose tissue-derived stromal cells-basic and clinical implications for novel cell-based therapies. Stem Cells, 25: 818-827.

Schipper, B.M., K.G. Marra, W. Zhang, A.D. Donnenberg and J.P. Rubin, 2008. Regional Anatomic and age effects on cell function of human adipose-derived stem cells. Ann. Plast. Surg., 60: 538-544.

Spencer, N.D., R. Chun, M.A. Vidal, J.M. Gimble and M.J. Lopez, 2011. In vitro expansion and differentiation of fresh and revitalized adult canine bone marrow-derived and adipose tissue-derived stromal cells. Vet. J.,

Torres, F.C., C.J. Rodrigues, I.N. Stocchero and M.C. Ferreira, 2007. Stem cells from the fat tissue of rabbits: an easy-to-find experimental source. Aesthetic Plast. Surg., 31: 574-578.

Toyoda, M., Y. Matsubara, K. Lin, K. Sugimachi and M. Furue, 2009. Characterization and comparison of adipose tissue-derived cells from human subcutaneous and omental adipose tissues. Cell Biochem. Funct., 27: 440-447.

Van Harmelen, V., K. Rohrig and H. Hauner, 2004. Comparison of proliferation and differentiation capacity of human adipocyte precursor cells from the omental and subcutaneous adipose tissue depot of obese subjects. Metab. Clin. Exp., 53: 632-637.

Vidal, M.A., G.E. Kilroy, M.J. Lopez, J.R. Johnson, R.M. Moore and J.M. Gimble, 2007. Characterization of equine adipose tissue-derived stromal cells: adipogenic and osteogenic capacity and comparison with bone marrow-derived mesenchymal stromal cells. Vet. Surg., 36: 613-622.

Williams, K.J., Picou A.A., S.L. Kish, A.M. Giraldo, R.A. Godke and K.R. Bondioli, 2008. Isolation and characterization of porcine adipose tissue-derived adult stem cells. Cells Tissues Organs., 188: 251-258.

Yamamoto, N., H. Akamatsu, S. Hasegawa, T. Yamada and S. Nakata et al., 2007. Isolation of multipotent stem cells from mouse adipose tissue. J. Dermatol. Sci., 48: $43-52$.

Zuk, P.A., M. Zhu, H. Mizuno, J. Huang and J.W. Futrell et al., 2001. Multilineage cells from human adipose tissue: Implications for cell-based therapies. Tissue Eng., 7: 211-228.

Zuk, P.A., M. Zhu, P. Ashjian, D.A. De Ugarte, J.I. Huang and H. Mizuno et al., 2002. Human adipose tissue is a source of multipotent stem cells. Moll. Biol. Cell, 13: 4279-4295. 